\title{
SHEAR-LAYER INSTABILITY IN A ROTATING SYSTEM
}

\section{S. Poncet and M. P. Chauve}

Institut de Recherche sur les Phénomènes Hors Equilibre (IRPHE), UMR CNRS 6594, Technopôle Château Gombert, 49 rue F. Joliot-Curie, B.P.146, 13384 Marseille Cedex 13 -France. (poncet@irphe.univ-mrs.fr)

The shear-layer instability in the flow over a rotating disk with a free surface is investigated experimentally by flow visualizations for a large range of the flow control parameters: the aspect ratio $G$ of the cavity, the rotationnal Reynolds number Re and the radius ratio s between the inner and outer radii of the rotating disk. This instability develops along the cylindrical shroud as sharp-cornered polygonal patterns characterized by the number of vortices $m$. This number $m$ can be scaled by considering an Ekman number based on the water depth, which confirms that the shroud boundary layer is of Stewartson type. The appearance threshold of the first polygonal mode is constant by considering the mixed Reynolds number introduced by Niino and Misawa (1984) based on both the water depth at rest and the rotating disk radius. For large values of s, the instability patterns appear along the hub as small stationary cells.

Keywords: shear-layer instability, rotating disk, flow visualization. 


\section{INTRODUCTION}

The stability of rotating disk flows has been addressed for a long time, mostly in the case of a single infinite rotating disk (Faller 1991) and more recently in the rotor-stator configuration (Cros et al. 05, Poncet \& Chauve 05). The present work considers an experimental system where steady modes of a shear-layer instability are obtained over a finite rotating disk with a free surface. Shear layers in rapidly rotating systems (called Stewartson layers) are of primary importance from a geophysical point of view, as they can be observed in oceans or in planetary atmospheres. For examples, the Great Red Spot on Jupiter and the Blue spot on Neptune result from differential rotation with sharply sheared zonal flows. Rotating shear layer instabilities have been seen also in flows enclosed in computer hard drives. The instability that affects a circular shear-zone has then been widely studied in various configurations.

Stewartson (1957) considered the stationary linear problem in the case of a split rotating cylinder. He showed, using an asymptotical method, that the flow generated by a slight differential rotation of one part of the shell is composed by two cylindrical shear layers aligned with the axis of rotation and located at the split radius. The largest one is geostrophic and its width scales like $E_{b}^{1 / 4}$ (where $E_{b}=v /\left(\Omega b^{2}\right)=1 /$ Re is the Ekman number based on the cylinder radius $b, v$ the kinematic viscosity of the fluid, $\Omega$ the rate of rotation of the cylinder and $R e$ the rotationnal Reynolds number), whereas the width of the thinner ones scales like $E_{b}^{1 / 3}$.

Hide and Titman (1967) investigated experimentally the linear stability of the Stewartson layers, which develop on a differentially rotating disk suspended in a rotating tank. The instability appears as non-axisymmetric patterns of $m$ waves in planes perpendicular to the 
rotation axis above a well-defined threshold. The number $m$ is maximum at the threshold and decreases with the amplitude of the differential rotation. In their experiments, the Coriolis force plays an important role in the development of the vortices proving that this is more than a simple Kelvin-Helmholtz instability. Rabaud and Couder (1983) performed experiments on the destabilization of a thin layer of air between two plates. The instability is of KelvinHelmholtz type and induces regular, steady patterns of $m$ vortices. Neither the centrifugal nor the Coriolis forces are involved in the motion. The same configuration has been considered later by Chomaz et al. (1988), who compared the results presented in Rabaud and Couder (1983) with new experiments and numerical simulations. They showed in particular the dependence on the aspect ratio of the cell on the dynamical behavior of the flow. In large cells, transitions from a mode with $m$ vortices to a mode with $(m-1)$ vortices occur through localized processes. On the contrary, in small cells, transitions occur after a series of bifurcations corresponding to successive breakings of all the symmetries of the flow. The same configuration has been studied numerically by Bergeron et al. (2000). They compared the results of their numerical simulation code with the experimental data and the linear stability analysis of Rabaud and Couder (1983) and Chomaz et al. (1988) with a reasonable agreement between the different approaches, although Bergeron et al. (2000) found a clear dependence of the critical Reynolds number on the aspect ratio of the shell.

Niino and Misawa (1984) considered a flow driven by a thin disk at the bottom of a tank to study the barotropic instability of horizontal shear flows. They compared their experimental results with a linear stability analysis applied to an initially circular shear-layer, including viscous diffusion and Ekman pumping. They found that the number of vortices should decrease with increasing Reynolds number. In a laboratory experiment, Früh and Read (1999) studied also the barotropic shear layer in a rotating fluid. Above a critical shear, the shear layer breaks up through barotropic instability, which appears as a string of vortices along the 
shear zone. They showed that the transition from the axisymmetric mode to regular vortices occurs through a Hopf bifurcation. They obtained modes $m$ in the range 2-8. Van de Konijnenberg et al. (1999) investigated experimentally and numerically the instability of a forced, circular shear layer in a rotating fluid. They applied a radial pumping to a shallow layer of water in a parabolic tank to model a geophysical $\beta$-effect. The instability appears as a sequence of vortices, the number of which decreases with increasing strength of the shear. The radial pumping of fluid from the periphery to the center of the cavity induces an azimuthal flow, which stabilizes the shear flow if it is opposite to the rotation or destabilizes it otherwise. A $\beta$-effect may prevent the formation of a steady vortex chain.

Dolzhanskii et al. (1992) considered a MHD device for the generation of both a circular shear flow and a Kolmogorov flow. They compared their experimental results to the stability theory of quasi-two-dimensional flows with a close agreement between the different approaches. In the circular geometry, the modes $m=3,5$ and 6 of the shear layer instability have been observed.

The flow between an enclosed corotating disk pair (ECDP) offers a well-known configuration to observe polygonal patterns. Abrahamson et al. (1989) studied experimentally this type of flow for a radius ratio $s=a / b=0.5,1.5 \times 10^{5} \leq \operatorname{Re} \leq 1.5 \times 10^{6}$ and $0.013 \leq G=h / b \leq 0.1$ ( $a$ and $b$ are respectively the inner and outer radii of the rotating disks and $h$ is the interdisk space). They observed three distinct regions: a solid body inner region near the hub, an outer region dominated by large counter-rotating vortices and a boundary layer along the shroud, the last one is three-dimensional (3D) contrary to the two others. They considered two flow control parameters: the Ekman number $E_{b}$ and the aspect ratio of the cavity $G$. When $E_{b}$ decreases or $G$ increases, the number of vortices $m$ decreases but they become larger. Herrero et al. (1999) established numerically the bifurcation diagram in the ECDP case in the plane $(R e, G)$ for $s=0.537, \operatorname{Re} \leq 82380$ and $0.05 \leq G \leq 0.2$. They 
distinguished three regions corresponding respectively to axisymmetric steady flow with interdisk midplane symmetry, to 3D unsteady flow with shift-and-reflect symmetry and to 3D unsteady flow with symmetry breaking with respect to the midplane. In the last case, they obtained the mode $m=4$ for $\operatorname{Re}=13710$ and $G=0.18$ and the mode $m=5$ for $\operatorname{Re}=82380$ and $G=0.091$ in agreement with the observations of Abrahamson et al. (1989). Randriamampianina et al. (2001) presented a numerical investigation of the flow in the ECDP case for $s=0.5,0.1 \leq G \leq 0.6$ and $\operatorname{Re}=1.06 \times 10^{4}$. For $G \geq 0.26$, they showed that the transition to unsteady $3 \mathrm{D}$ flow occurs after the pitchfork bifurcation. The flow structure is then characterized by a shift-and-reflect symmetry, which is consistent with the experiments of Abrahamson et al. (1989). For smaller values of the aspect ratio, the 3D flow shows a symmetry breaking. For $G=0.6$ and increasing values of the rotation rate, they obtained successively the mode $m=8$ for $\operatorname{Re}=3750$, the mode $m=6$ for $\operatorname{Re}=4250$ and the mode $m=5$ for $\operatorname{Re}=10^{4}$.

The shear layer instability in the flow between two counter-rotating disks enclosed by a cylinder has been investigated experimentally and numerically by Moisy et al. (2004) for $s=0$ and $0.048 \leq G \leq 0.5$. It develops as a sharp-cornered polygonal pattern with $m$ sides, already observed by Lopez et al. (2002), surrounded by a set of $2 \mathrm{~m}$ spiral arms. The lower modes $m=3-5$ are observed essentially for large aspect ratios and the number of sides increases for decreasing values of the aspect ratio. No hysteresis is reported for the instability threshold but a noticeable one is present for the onset mode. The authors believe that the bifurcation remains supercritical for the whole range of the flow control parameters. The reader is referred to the work of Moisy et al. (2004) for a more extensive literature survey on such a flow configuration.

The reader is referred also to the review paper of Dolzhanskii et al. (1990) and to the work of Bergeron et al. (1996) for discussion about the analysis of the shear layer with weakly 
nonlinear theory, which accounts for phenomena such as hysteresis and saturation of the amplitude of the unstable wave mode.

\section{EXPERIMENTAL SET-UP AND FLOW CONTROL PARAMETERS}

\subsection{The apparatus}

A sketch of the experimental cell is presented in figure 1. It consists of a smooth rotating stainless-steel disk of radius $b=140 \mathrm{~mm}$ enclosed by a fixed cylindrical shroud of radius $b+j=140.85 \mathrm{~mm}(j=0.85 \pm 0.05 \mathrm{~mm})$. A central hub of radius $a$ equal to either 40,75 or $105 \mathrm{~mm}$ can be attached or not $(a=0)$ to the rotating disk. The disk drive shaft is going through the bottom of the tank and is connected to an electric engine by means of a belt, so that the disk can rotate with an angular velocity $\Omega$ varying from 0 to $200 \mathrm{rpm}$. A servo-contro system for the rotation rate permits to maintain $\Omega$ constant with an accuracy of $0.2 \%$. Note that both the disk and the hub rotate clockwise. The heights of the cylinder and the hub are fixed to $h=20 \mathrm{~mm}$. The cavity is filled up by water at constant working temperature $20^{\circ} \mathrm{C}$ (kinematic viscosity of water $v=10^{-6} \mathrm{~m}^{2} / \mathrm{s}$ ). The water depth at rest is denoted $e$ and can vary between 0 and $15 \mathrm{~mm}$.

In order to visualize the hydrodynamic structures, which develop in the flow, the water is seeded with reflective anisotropic particles of "kalliroscope" (size $30 \times 6 \times 0.07 \mu \mathrm{m}$ ) in suspension, whose orientation depends upon the shear stress of the flow. We illuminate the flow with an annular neon and the surface of the stainless steel rotor is painted black to improve the visualizations. Images $(768 \times 576$ pixels $)$ are taken at a video frequency of 25 
images per second using a CCD video camera situated one meter above the cavity. The camera can rotate to study instabilities in their reference frame.

\subsection{The parameters}

The flow is mainly controlled by three control parameters: the rotationnal Reynolds number Re based on the outer radius $b$ of the rotating disk, the aspect ratio $G$ of the cavity and the radius ratio $s$ defined as followed:

$$
\operatorname{Re}=\frac{\Omega b^{2}}{v} \leq 1.2 \times 10^{5} \quad G=\frac{e}{b} \leq 0.107 \quad s=\frac{a}{b}=0,0.286,0.536,0.75
$$

Note that $s=0$ corresponds to the case where no hub is attached to the rotating disk. These dimensionless numbers appeared to be the more common ones as they contain only one varying parameter.

The axial $z^{*}$ and $r^{*}$ radial dimensionless coordinate are defined as followed: $z^{*}=z / H$ and $r^{*}=r / b$, where $H(r)$ is the local water depth (see next section). $z^{*}=0$ corresponds to the rotating disk surface and $z^{*}=1$ to the free surface.

For future discussions, the Ekman number based on the water depth $e$ at rest is introduced: $E_{e}=v /\left(\Omega e^{2}\right)$. It measures the viscous dissipation compared to the Coriolis term. Numerous authors introduced also a Rossby number Ro based on the differential rotation of their system. In the present work, the shear layer is produced by the differential rotation between the rotating fluid $\left(\Omega_{f}\right)$ and the fixed shroud $\left(\Omega_{s}=0\right)$. The Rossby number Ro based on the differential rotation $\left(\Omega_{f}-\Omega_{s}\right) / \Omega_{f}$ is then equal to $R o=1$ and does not appear to be a relevant parameter to study the stability of this flow. 


\subsection{The experimental procedure}

We have observed that the stability of the basic flow is very sensitive to the initial conditions (in particular to the initial shear stress), but also to the time history of the rotation rate as it is the only varying flow control parameter during a sequence of observations. Thus, the thresholds and the sequence of the instabilities depend strongly on the experimental procedure, which must be always the same. In order to impose a "continuous" shear between the rotating fluid and the shroud, we have chosen to increase the rotation rate $\Omega$ by step of one rpm between two observations. According to the linear Ekman dynamics (Greenspan, 1968), the time evolution in rotating flow systems is characterized by the Ekman time: $\tau_{E}=b / \sqrt{(1 \Omega)} \approx 137 \mathrm{~s}$ for $\Omega=10 \mathrm{rpm}$. As long as the symmetry (the number of vortices) is constant, the adjustment of the flow to changes in $R e$ is almost instantaneous (Bergeron et al. 2000) but the time scale related to the transition between two modes is much longer and close to $\tau_{E}$. As soon as the last pattern is obtained for large values of the rotation rate, the same procedure is applied for decreasing values of $\Omega$ until the last mode disappears. The shear is supposed to be constant along the axial direction as the water depth $e$ remains small. All results reported in the next section were found to be repeatable. For future discussions, as already introduced by Bergeron et al. (2000), spin up (resp. spin down) refers to a sequence of observations where the values of the Reynolds number $R e$ are increased (resp. decreased).

\subsection{Shape of the free surface for $s=0$}

The experiments were performed in a shallow layer of water over a rotating disk. The radial and axial pressure gradients are respectively given by the centrifugal force and the 
gravity. Thus, the shape of the free surface is parabolic and the water depth depends on the radial location $r$. The local water depth $H(r)$ can be expressed for $s=0$ as:

$$
H(r)=e+\frac{\Omega_{f}^{2}}{2 g}\left(r^{2}-\frac{b^{2}}{2}\right)
$$

where $g=9.81 \mathrm{~m} / \mathrm{s}^{2}$ is the acceleration of gravity. The fluid velocity $\Omega_{f}$ has been measured by laser Doppler anemometry (LDA). It rotates almost as a solid body with a tangential velocity directly proportionnal to the rotating disk speed: $\Omega_{f}=K \Omega$, with $K$ the entrainment coefficient of the fluid equal to $0.84 \pm 0.05$ depending slightly on both the spatial location and the radius ratio. This value of $\mathrm{K}$ is close to the one obtained by Herrero et al. (1999) in the ECDP case: $K \approx 0.85$. We have experimentally verified equation (1) for different values of $\Omega$. For the range of parameters considered in the present work ( $\Omega \leq 59 \mathrm{rpm}, e \leq 15 \mathrm{~mm})$, the water depth $H(r)$ is always larger than the Ekman layer thickness $\delta=\sqrt{v / \Omega}$, which implies that the centrifugal and Coriolis forces may have an influence on the flow. The gradient in the depth of the fluid layer induces a similar effect to the $\beta$-effect encountered in atmospheric and oceanic flows (see Van de Konijnenberg et al. 1999), with $\beta=2 \Omega_{f} H^{-1} d H / d r$. As already mentionned, the Rossby number Ro being equal to one, the topographic $\beta$-effect is weak in the present case. Moreover, Manin (1990) and Van de Konijnenberg et al. (1999) reported that it does not affect the dependence of the number of vortices on the Reynolds number.

\section{RESULTS AND DISCUSSION}

\subsection{Structure of the flow for $s=0$}


Figure 2 illustrates an example of the flow structure (mode $m=5$ ) observed for $s=0$, $\operatorname{Re}=45155$ and $G=0.0429$. This illustration is quite comparable to the vorticity maps obtained by direct numerical simulations (see for example Randriamampianina et al 2001) in the $(r, \theta)$ plane, where $r$ and $\theta$ are respectively the radial and tangential directions. The vortical structure occupies all the water depth as the shear is supposed to be constant along the axial direction (small values of $G$ ). We can distinguished three distinct radial regions, the inner and outer regions, separated by a polygonal boundary.

The inner region is the region observed for $0 \leq r^{*}=r / b \leq 0.64$, in which the flow is twodimensional, laminar and rotates roughly as a solid body. The shape of the inner region is polygonal with $m=5$ sides. During flow visualization in the rotating frame of reference, we observed that the polygon rotates slightly relative to the rotating disk, which is induced by the passage of the vortical structures in the outer region.

The outer region, confined between the inner region and the shroud, is actively turbulent. It contains $m=5$ counterclockwise vortical structures. These structures are almost distributed along a circle of radius $r_{c}^{*}=r_{c} / b$, with $r_{c}=\left(R_{1}+R_{2}\right) / 2 . R_{1}$ and $R_{2}$ are respectively the minimum radial location of the polygonal boundary and the maximum radial location of the vortical structures as shown in figure 2. This critical radial location for the appearance of the polygonal patterns is approximately equal to $r_{c}^{*} \approx 0.73$

The five vortices, which span the radial extent of the outer region, rotate relatively to the rotating disk with a speed, estimated from the flow visualization, of about $79 \%$ of the disk speed relative to the laboratory. This value is in good agreement with the $75 \%$ obtained by Abrahamson et al. (1989) for $m=5, G=0.05$ and $\mathrm{Re}=4.924 \times 10^{5}$.

The vortical structures are responsible for the deformation of the inner region, which produces the polygonal boundary. As showed by the straight arrows in figure 2, the portion of 
the vortex that flows outward induces the lobes of the polygon by pulling the inner region fluid away from the center of the rotating disk. In the same way, the portion of the vortex that flows inward (dashed arrows) flattens the boundary by forcing it radially inward. Thus, the shape of the boundary moves with the vortices. The inner region fluid moves with the vortices too. In response to the passing train of vortices, it moves slightly in cyclic orbital motion.

At the periphery of the rotating disk, a boundary layer develops along the fixed cylindrical shroud. This shroud boundary layer is three-dimensional according to the observations of Abrahamson et al. (1989).

\subsection{Transition diagram for $s=0$}

The main results concern a cavity where no hub is attached to the rotating disk $(s=0)$. The two only flow control parameters are the water depth $e(G=e / b)$ and the rotation rate $\Omega$ ( $\left.\operatorname{Re}=\Omega b^{2} / v\right)$. The purpose to the two next subsections is to provide flow visualizations of the shear instability and transition diagrams for various geometries. The reader is referred to the numerical studies of Chomaz et al. (1988) and Bergeron et al. (2000) for a detailed description of the transition processes during spin-up and spin-down, which is quite difficult to report from experimental observations.

In figures 3 to 5 , we represent the stability diagram of the basic flow in the plane $(m, R e)$ for three characteristic aspect ratios. We recall that $m$ is the number of vortices. For $G=0.0714$ (figure 3), the flow becomes unstable above a first threshold $\operatorname{Re}=4105$ and the axisymmetric mode denoted $m=0$ appears as a first circle $\mathrm{C} 1$ (figure 6a) moving slightly towards the center of the rotating disk for increasing values of the Reynolds number. A second circle C2 appears along the shroud and moves also towards the center of the disk. The flow is then almost entirely laminar. When both circles interact for $\operatorname{Re}=16420$ (it 
corresponds to $\operatorname{Re}_{N M}$ introduced below), a second pattern develops as a sharp cornered polygon with five vortices (see figure 6e). When the Reynolds number is further increased above this critical value, a series of two symmetry-breaking bifurcations occur. Each one of these is characterized by a reduction of the number of vortices in the system. The transition from the mode $m=5$ to the mode $m=4$ (figure $6 \mathrm{f}$ ) occurs above a third threshold $\mathrm{Re}=36945$. The existence domain of the mode $m=4$ is quite narrow, contrary to the mode $m=3$ (figure $6 \mathrm{~g}$ ), which subsists in the range $43103 \leq \mathrm{Re} \leq 102625$. For larger Reynolds numbers, an axisymmetric state (figure 6h) is restored but the flow at the periphery of the cavity is turbulent and the mode denoted $m=1$ appears. During spin-down (dashed arrows in figure 3), only the modes $m=1,3,5$ and 0 are successively obtained. The sequence has then changed and the thresholds are slightly shifted to smaller values of $R e$ than in the case of spinup. Finally the flow becomes again stable for $\mathrm{Re}=4105$. During spin-down, the number of vortices in the flow has increased by more than one when the transition from mode $m=3$ to mode $m=5$ occurred. It could be assigned to a too fast spin-down or to an external noise, which postpones the transition as suggested by Bergeron et al. (2000).

Note that the mode $m=2$ has never been observed for $s=0$ and that the modes $m=0$ and 1 are always obtained whatever the geometry of the cavity (all values of $G$ and $s$ ). All the observations reported here are in good agreement with other experiments (Chomaz et al. 1988, Moisy et al. 2004), where strong hysteresis is observed for the modes, although no or a weak hysteresis is present for the threshold values. This problem is generic for systems where the geometrical confinement leads to azimuthal wavenumber quantization (Rabaud \& Couder 1983).

When the aspect ratio $G$ decreases (figures 3 to 5 ), the number of vortices of the first polygonal pattern increases: $m=5$ for $G=0.0714$ (figure 3), $m=6$ for $G=0.0429$ (figure 4) and $m=8$ for $G=0.0179$ (figure 5). The sequence is almost the same whatever the aspect 
ratio. $N$ polygonal patterns are observed during the spin-up and $(N-1)$ patterns during the spindown. The number of observed polygonal structures $N$ decreases from $N=3$ for $G=0.0719$ and $G=0.0429$ to $N=2$ for $G=0.0179$. The instability thresholds are also slightly shifted to smaller values of the Reynolds number when $G$ decreases. Note that the hysteresis on these thresholds is more important for the smallest value of $G=0.0179$ (figure 5). Moreover for this aspect ratio, the transition process during the spin-down does not continue until the same number of vortices obtained at the threshold is reached. This result is in contrast with the numerical simulations of Bergeron et al. (2000), which is probably due to both the configuration and especially to the experimental procedure.

Figure 7 presents the marginal stability diagram of the first observed polygonal mode in the plane $(R e, G)$ for $s=0$ and increasing values of the Reynolds number Re. For large aspect ratios $G$, the first polygonal pattern appears for small values of $R e$ and the number of vortices $m$ is then small. When $G$ decreases, $R e$ and $m$ increase. For $s=0$, modes in the range $m=4-8$ have been obtained for $G \leq 0.12$.

The first observed polygonal mode $m$ (during spin-up) can be scaled by: $m \sim E_{e}^{-1 / 4}$ as already mentioned by Schaeffer and Cardin (2005) in their study of the instabilities of the Stewartson layer in flat and depth-varying containers or by Moisy et al. (2004) in the flow between counter-rotating disks, where $E_{e}=v /\left(\Omega e^{2}\right)$ is the Ekman number based on the water depth $e$ at rest. The reader is referred also to the brief review of Manin (1990) for similar behaviors and to the pioneering work of Stewartson (1957). Figure 8 showed indeed that, in the present case, $m \sim-2.26 \times E_{e}^{-1 / 4}$. It confirms that the boundary layer along the shroud is a Stewartson boundary layer, whose thickness is given by $E_{e}^{1 / 4} b$.

From figures $6 \mathrm{~b}$ to $6 \mathrm{~g}$, we can deduce the critical radius location for the appearance of the polygonal patterns: $r_{c}^{*}=r_{c} / b$, with $r_{c}=\left(R_{1}+R_{2}\right) / 2$ (see figure 2). It appears from all the 
observations that this critical radius is almost constant whatever the number of vortices $m$ : $r_{c}^{*} \approx 0.73$. Note that it is not the case for the minimum radial location of the polygonal boundary $R_{1}$ and for the maximum radial location of the vortical structures $R_{2}$.

Niino and Misawa (1984) proposed a Reynolds number, denoted here $\operatorname{Re}_{N M}=V L / v$, as the only parameter which governs the stability of the basic flow, where $V$ and $L$ are the characteristic velocity and length of the basic flow. They found a critical Reynolds number $\operatorname{Re}_{N M} \approx 11.7 \pm 0.5$. Note that Manin (1990) mentionned that the value of $\operatorname{Re}_{N M}$ depends on the axial profile of the radial velocity. In the present case, $V$ is defined by $V=\Omega b$ as the shear is produced at the periphery of the rotating disk, and the thickness $L$ of the shear layer is given by $L=\left(E_{e} / 4\right)^{1 / 4} \times e$ (Niino \& Misawa, 1984). Thus, $\operatorname{Re}_{N M}$ can be expressed as: $\operatorname{Re}_{N M}=b e^{1 / 2}(\Omega / v)^{3 / 4} / \sqrt{2}$. We have verified, in figure 9, that the critical Reynolds number $\operatorname{Re}_{N M}$ is almost constant: $\operatorname{Re}_{N M} \approx 62$. This value is an estimate since the instability takes place for radii lower than the cell radius $b$. It confirms that, as the Rossby number is close to 1 , the Reynolds number $\operatorname{Re}_{N M}$ is the only preponderant parameter to study the stability of the basic flow. It means also that the ratio $\mathrm{m} / \delta$ between the number of vortices and the Ekman layer thickness is constant.

Spiral patterns have also been observed. They can coexist with the polygonal structures as they are located at the periphery of the rotating disk as shown in figure 10. As the vortical structures, a weak hysteresis is observed on the thresholds of the instability (tab.1). They appear for relatively small values of the Reynolds number. Figures 11 and 12 represent two space-time diagrams respectively in terms of an angle $\theta^{*}=\theta /(2 \pi r)$ (at $r^{*}=0.95$ ) and in terms of a radius $r^{*}$ of the flow for $s=0, R e=24630$ (spin-up) and $G=0.0714$. They correspond to the flow visualization in figure 10 in the frame of reference of the polygonal 
pattern. We define the dimensionless time as $t^{*}=2 \pi t / \Omega$. The diagonal lines in figure 11 correspond to the spiral passing. The negative slope indicates that they move slower than the vortices $(16.6 \%$ of the passing velocity of the polygonal structures ie $0.125 \Omega r$ ). The spacetime diagram according to a radius (figure 12) shows the radial extent of the spiral rolls, their inclination angle $\varepsilon$ and their azimuthal wavenumber $n$, which is also the number of structures by rotation. We can notice also the passing of the vortical structures. The characteristics of the spirals are sumed up in table 1 . Note that they have not been observed for $G=0.0179$. These are positive spiral patterns as they are rolled up towards the disk axis in the rotation sense of the rotor. The inclination angle remains small $14 \leq \varepsilon \leq 25^{\circ}$ compared to the values obtained by Poncet and Chauve (2005) in a rotor-stator cavity with throughflow ( $\varepsilon \rightarrow 70^{\circ}$ ), as well as the number of structures $28 \leq n \leq 40$ to be compared to $n \rightarrow 90$. As already mentionned, they are located at the periphery of the cavity for $r^{*} \geq 0.85$. All these characteristics are close to the ones of the SRJ2 spirals studied by Poncet and Chauve (2005) in the case of weak inward throughflow. These authors showed that it is a crossflow instability due to the inflexion point in the axial profile of the mean radial velocity. It can be noticed that the characteristics of the spirals slightly depend on the aspect ratio of the cavity $G$. In fact, the value of $G$ (and the one of $R e$ ) settles the number of vortices, which settles itself the characteristics of the SRJ2 rolls.

\subsection{Influence of the radius ratio $s$}

Four values of the radius ratio $s$ have been considered: $s=0,0.286,0.536$ and 0.75 . Figures 4,13 and 14 present the stability diagrams of the flow for $G=0.0429$ and three values of the radius ratio in the plane $(m, R e)$. Whatever the value of $s \leq 0.536$, modes in the range $m=4-6$ have been obtained but the thresholds and the sequences are different. When 
the size of the hub increases, the radial extent of the inner region decreases strongly and the fluid rotates slightly faster, which settles the values of the thresholds. Note that for $s=0.536$ and $G=0.0714$, the mode $m=2$ (figure 15) has been observed in the range $32840 \leq \operatorname{Re} \leq 41050$ only during spin-down.

Figures 16a to $16 \mathrm{~d}$ represent the influence of the radius ratio $s$ on the flow pattern for $G=0.0429, \operatorname{Re}=36945$ (spin-up). Apart from the largest value of the aspect ratio $s=0.75$, the mode $m=6$ is observed. The critical radius $r_{c}^{*}$ is almost constant whatever the value of $s: r_{c}^{*} \approx 0.73$ for $s=0,0.286$ and 0.536 . When the radius ratio $s$ is larger than the value of the critical radius, the radial extent between the hub and the shroud is too small for the development of the vortical structures. Thus, for $s=0.75$ (fig.16d), the critical size of the system has been reached and no patterns are observed .

For $s=0.75$, no vortical structures are obtained but new stationary patterns develop along the hub as small cells (figures 17 a to 17 f) only for $G \geq 0.0714$. 10 cells appear above a first threshold $\operatorname{Re}=10263$. From $\operatorname{Re}=10263$ (figure $17 \mathrm{a}$ ) to $\mathrm{Re}=16420$ (figure $17 \mathrm{~b}$ ), the number of cells decrease from 10 to 9 but they grow both in the radial and tangential directions. They break up into 11 cells for $\mathrm{Re}=20525$ (figure 17c), then into 12 larger cells for $\operatorname{Re}=24630$ (figure $17 \mathrm{~d}$ ). From $\mathrm{Re}=24630$ (figure $17 \mathrm{~d}$ ) to $\mathrm{Re}=28735$ (figure $17 \mathrm{e}$ ), some cells merge and only 8 structures can be observed. When the Reynolds number is further increased, the number and the size of the patterns does not change but the flow confined between the cells and the shroud becomes turbulent (figure 17f) and is fully turbulent for $\mathrm{Re}=36945$. 


\section{CONCLUSION}

We have presented visualizations of the shear layer instability developed on a rotating disk with a free surface. Despite the different flow medias (gas, water, plasma) and the different forcing mechanisms producing the shear layer, the qualitative behavior of the flow is remarkably similar. For low Reynolds numbers, the flow is axisymmetric and becomes unstable above a first threshold $\operatorname{Re}_{N M}$ with a well-defined azimuthal mode number $m$. The instability appears as a sharp-cornered polygonal pattern with $m$ vortices.

The number of vortices of the first polygonal mode can be scaled by the Ekman number based on the water depth at rest to the power $-1 / 4$, showing that the shroud boundary layer is of Stewartson type (Schaeffer \& Cardin, 2005). The instability sets in at a well defined value of the Reynolds number based on the thickness of the shear layer: $\operatorname{Re}_{N M} \approx 62$. This supports the validity of the quasi-geostrophic approximation used in the theoretical work of Niino and Misawa (1984). The critical radial location for the appearance of the polygonal patterns is also

constant and equal to $r_{c}^{*} \approx 0.73$. At a given aspect ratio, when the Reynolds number $R e$ is increased (spin-up), these $m$ vortices transform into new arrangements with a decreasing number of vortices (until (m-2) in particular cases). For decreasing values of Re (spin-down), the flow demonstrates a noticeable hysteresis for the modes and a slight hysteresis for the thresholds, as already mentionned by Rabaud and Couder (1983). At a given rotation rate, the number of vortices increases for decreasing values of the water depth (or the aspect ratio). Note that modes up to $m=8$ have been obtained.

Finally, the influence of the radius ratio has been investigated. The development of the shear layer instability is constrained by the geometry of the system. When the size of the hub increases, the fluid rotates slightly faster, which shifts the values of the thresholds. Above the 
critical value of the radius ratio, new patterns are observed and develop along the hub as small stationary cells.

The authors would like to thank Dr Patrice Le Gal and Dr Nathanaël Schaeffer (IRPHE) for fruitful discussions.

\section{REFERENCES}

1. S.D. Abrahamson, J.K. Eaton and D.J. Koga, The flow between shrouded corotating disks, Phys. Fluids A, vol. 1 (2), pp. 241-251, 1989.

2. K. Bergeron, E.A. Coutsias, J.P. Lynov and A.H. Nielsen, Self-organization in circular shear layers, Physica Scripta, T67, pp. 33-37, 1996.

3. K. Bergeron, E.A. Coutsias, J.P. Lynov and A.H. Nielsen, Dynamical properties of forced shear layers in an annular geometry, J. Fluid Mech., vol. 402, pp. 255-289, 2000.

4. J.M. Chomaz, M. Rabaud, C. Basdevant and Y. Couder, Experimental and numerical investigation of a forced circular shear layer, J. Fluid Mech., vol. 187, pp. 115-140, 1988.

5. A. Cros, E. Floriani, P. Le Gal and R. Lima, Transition to turbulence of the Batchelor flow in a rotor/stator device, Eur. J. Mech. B/Fluids, vol. 24, pp. 409-424, 2005.

6. F.V. Dolzhanskii, V.A. Krymov and D.Y. Manin, Stability and vortex structures of quasitwo-dimensional shear flows, Sov. Phys. Usp., vol. 33 (7), pp. 495-520, 1990.

7. F.V. Dolzhanskii, V.A. Krymov and D.Y. Manin, An advanced experimental investigation of quasi-two-dimensional shear flows, J. Fluid Mech., vol. 241, pp. 705-722, 1992.

8. A.J. Faller, Instability and transition of disturbed flow over a rotating disk, J. Fluid Mech., vol. 230, pp. 245-269, 1991. 
9. W.G. Früh and P.L. Read, Experiments on a barotropic rotating shear layer. Part 1. Instability and steady vortices, J. Fluid Mech., vol. 383, pp. 143-173, 1999.

10. H.P. Greenspan, The Theory of Rotating Fluids, Cambridge University Press, 1968.

11. J. Herrero, F. Giralt and J.A.C. Humphrey, Influence of the geometry on the structure of the flow between a pair of corotating disks, Phys. Fluids, vol. 11 (1), pp. 88-96, 1999.

12. R. Hide and C.W. Titman, Detached shear layers in a rotating fluid, J. Fluid Mech., vol. 29, part 1, pp. 39-60, 1967.

13. J.M. Lopez, J.E. Hart, F. Marques, S. Kittelman and J. Shen, Instability and mode interactions in a differentially-driven rotating cylinder, J. Fluid Mech., vol. 462, pp. 383409, 2002.

14. D.Y. Manin, Characteristics Size of Vortices in Developed Quasi-Two-Dimensional Flows, Izvestiya, Atmospheric and Oceanic Physics, vol. 26, nº6, pp. 426-429, 1990.

15. F. Moisy, O. Doaré, T. Pasutto, O. Daube and M. Rabaud, Experimental and numerical study of the shear-layer instability between two-counter-rotating disks, J. Fluid Mech., vol. 507, pp. 175-202, 2004.

16. H. Niino and N. Misawa, An experimental and theoretical study of barotropic instability, J. Atmos. Sci., vol. 41, pp. 1992-2011, 1984.

17. S. Poncet and M.-P. Chauve, Crossflow instability in a rotor-stator cavity with axial inward throughflow, J. Fluid Mech., vol. 545, pp. 281-289, 2005.

18. M. Rabaud and Y. Couder, A shear-flow instability in a circular geometry, J. Fluid Mech., vol. 136, pp. 291-319, 1983.

19. A. Randriamampianina, R. Schiestel and M. Wilson, Spatio-temporal behaviour in an enclosed corotating disk pair, J. Fluid Mech., vol. 434, pp. 39-64, 2001.

20. N. Schaeffer and P. Cardin, Quasigeostrophic model of the instabilities of the Stewartson layer in flat and depth-varying containers, Phys. Fluids, vol. 17, 2005. 
21. K. Stewartson, On almost rigid rotations, J. Fluid Mech., vol. 3, pp. 17-26, 1957.

22. J.A. Van de Konijnenberg, A.H. Nielsen, J. Juul Rasmussen and B. Stenum, Shear-flow instability in a rotating fluid, J. Fluid Mech., vol. 387, pp. 177-204, 1999. 


\begin{tabular}{|c|c|c|c|c|c|}
\hline$G$ & $\operatorname{Re}($ spin-up) & $\operatorname{Re}($ spin-down) & $n$ & $\varepsilon\left(^{\circ}\right)$ & $r^{*}$ \\
\hline 0.0714 & $24630-43102$ & $16420-36945$ & $28-30$ & $23-25$ & $0.85-0.89$ \\
\hline 0.0429 & $30788-57470$ & $24630-45155$ & $38-40$ & $14-19$ & $0.88-0.92$ \\
\hline 0.0179 & - & - & - & - & - \\
\hline
\end{tabular}

Tab. 1. Characteristics of the SRJ2 spirals.

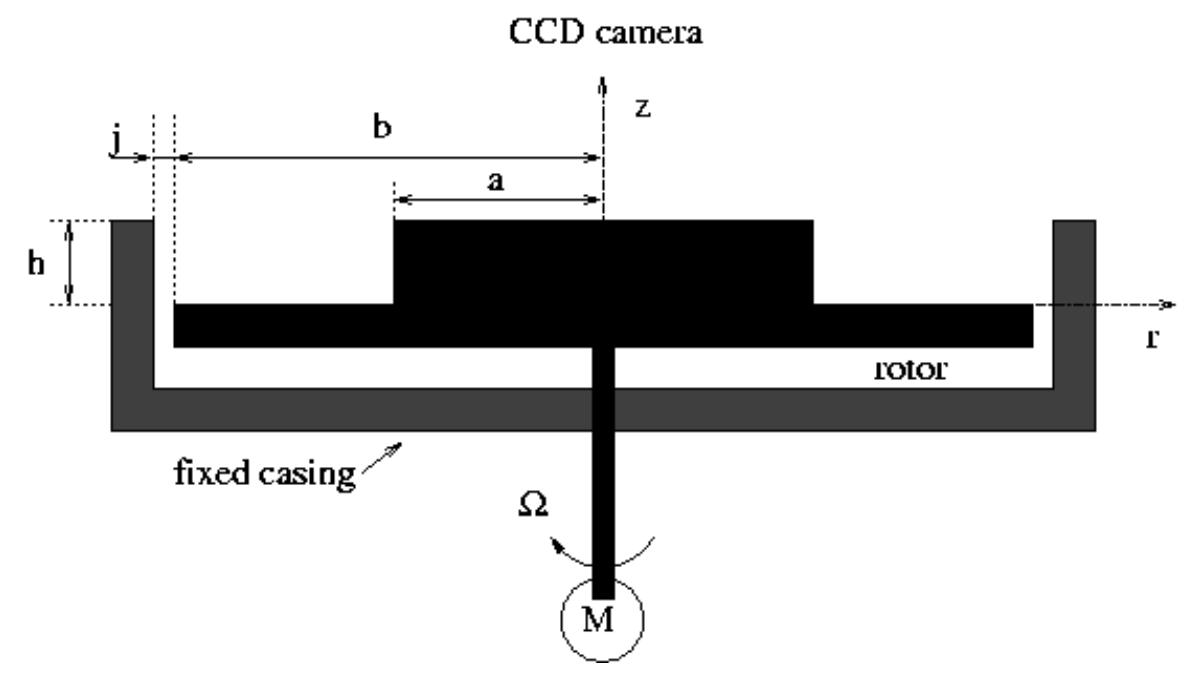

Fig. 1 Schematic of the experimental set-up with relevant notation. 


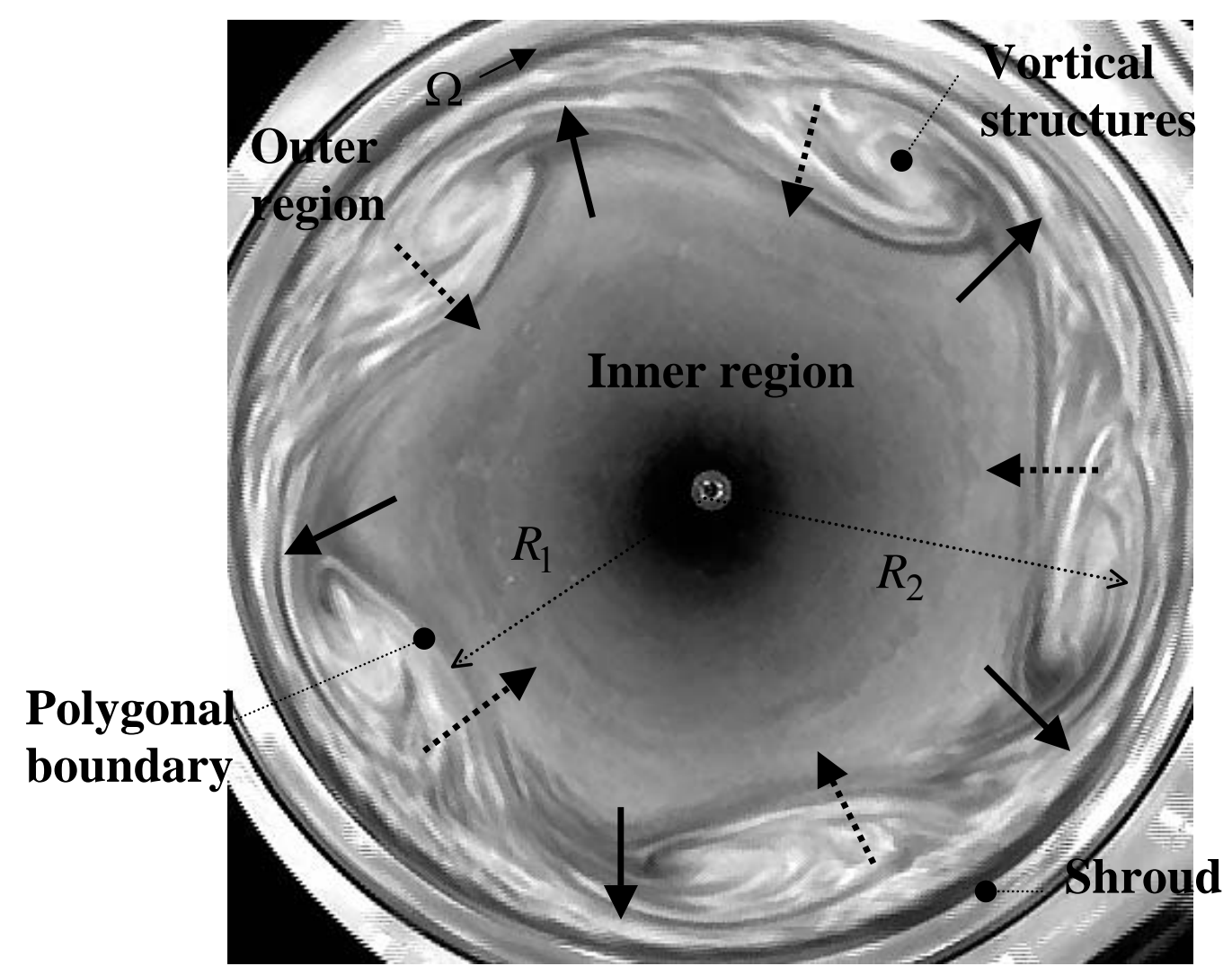

Fig. 2 Exemple of the flow structure (mode $m=5$ ) for $s=0, \operatorname{Re}=45155, G=0.0429$.

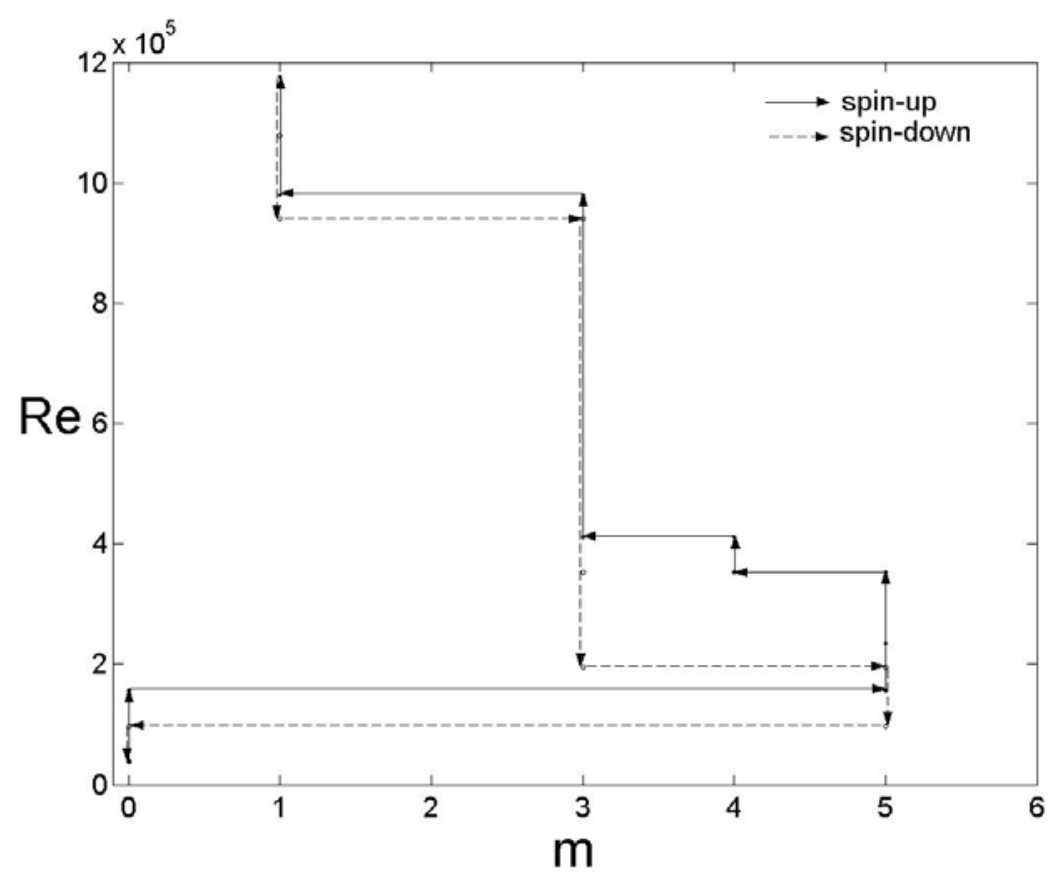

Fig. 3 Stability diagram for $s=0$ and $G=0.0714$. 


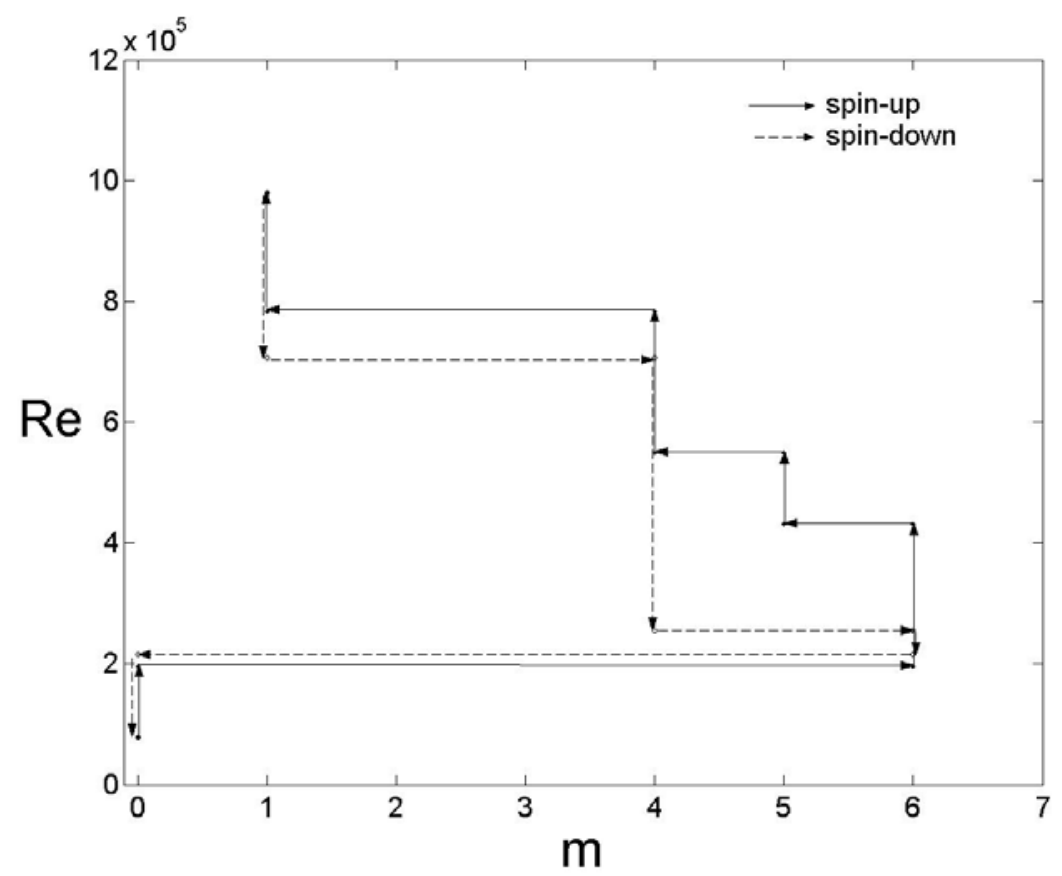

Fig. 4 Stability diagram for $s=0$ and $G=0.0429$.

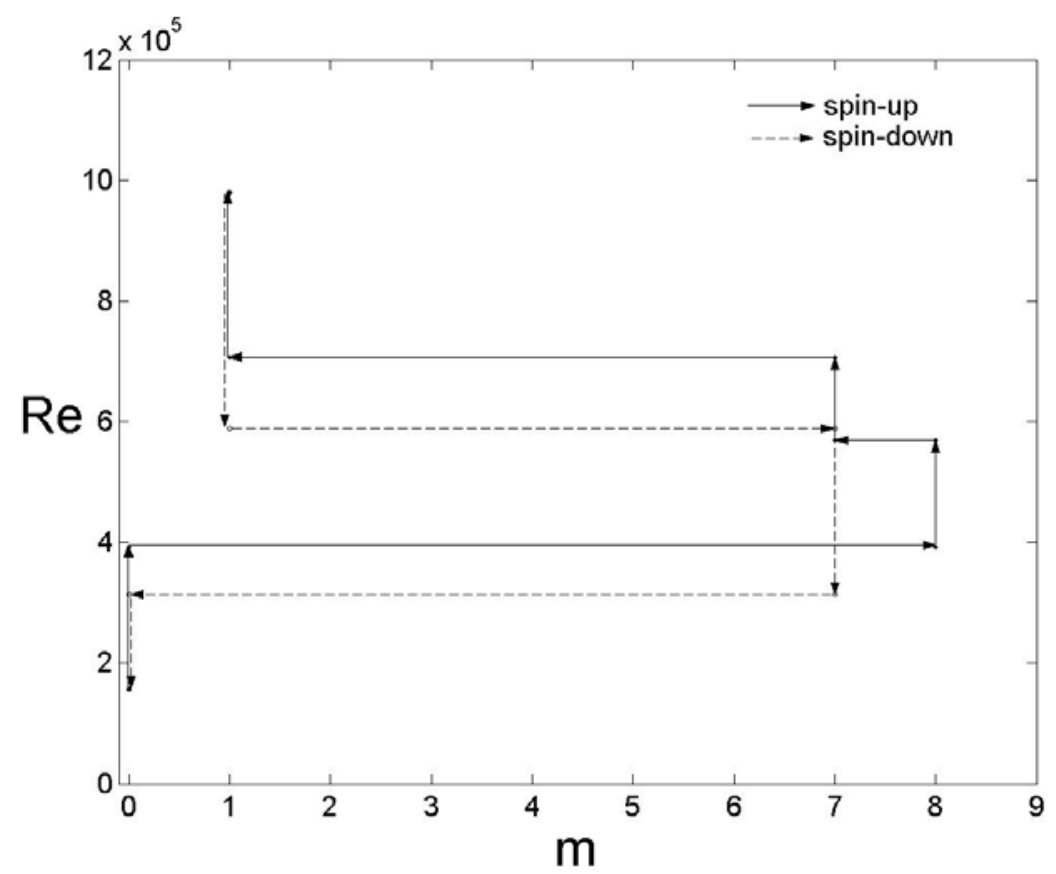

Fig. 5 Stability diagram for $s=0$ and $G=0.0179$. 
(a)

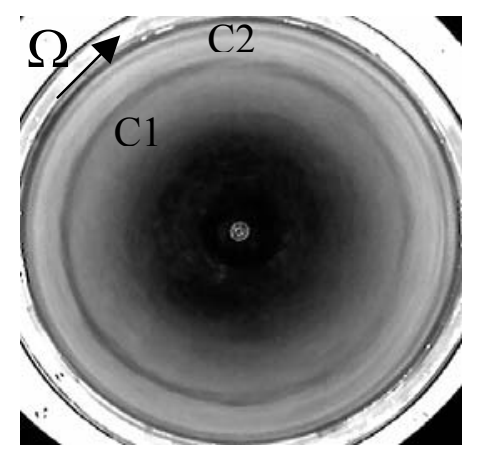

(d)

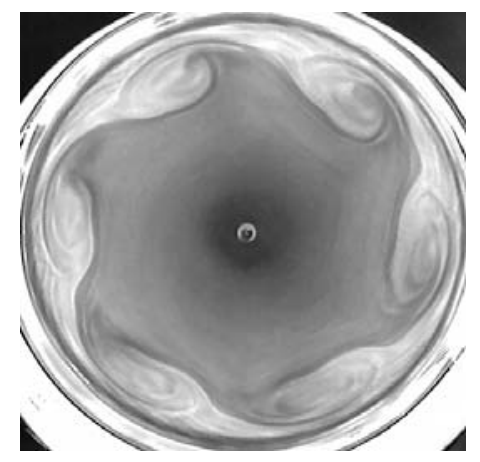

(g)

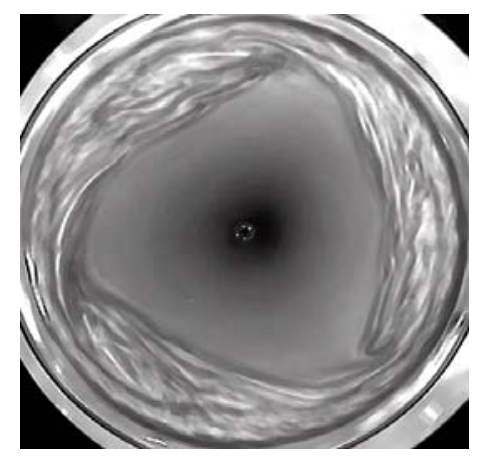

(b)

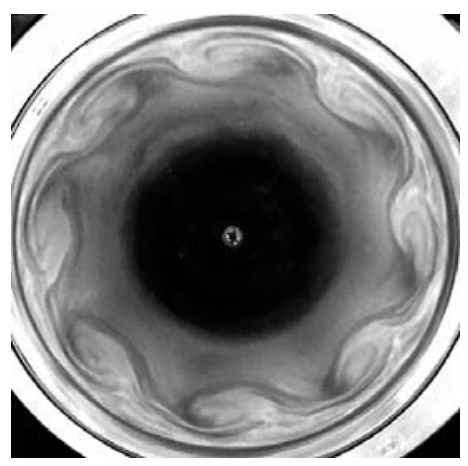

(e)

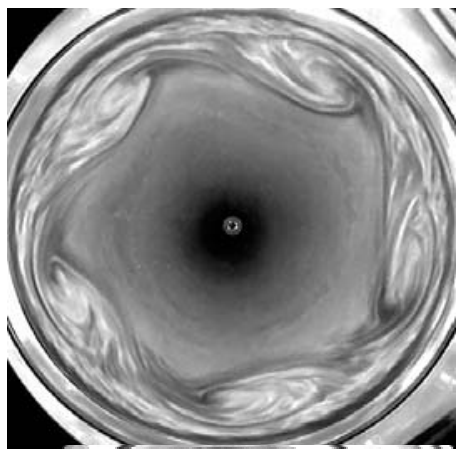

(h)

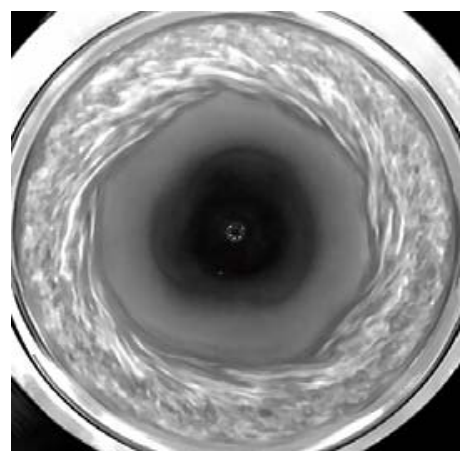

(c)

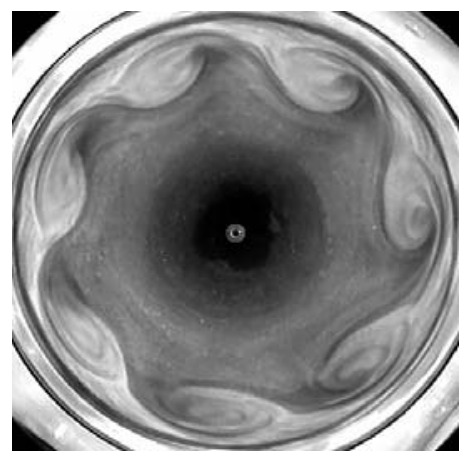

(f)

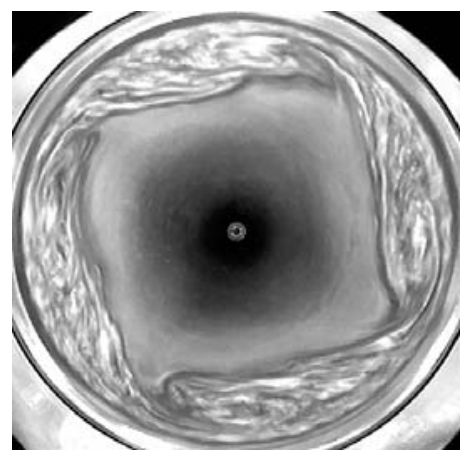

(i)

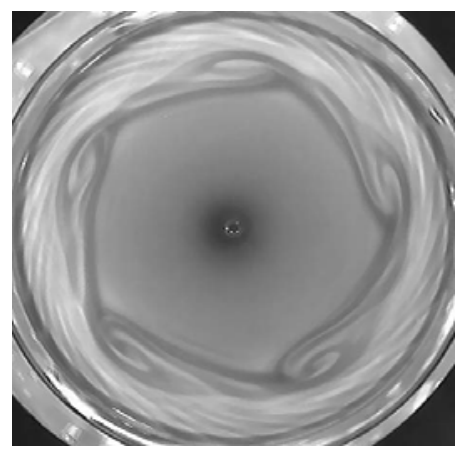

Fig. 6 Shear-layer instabilities for $s=0:$ (a) $m=0, G=0.0179, \operatorname{Re}=38998$,

(b) $m=8, G=0.0179, \operatorname{Re}=51313$, (c) $m=7, G=0.0286, \operatorname{Re}=43101$,

(d) $m=6, G=0.0429, \operatorname{Re}=34893$, (e) $m=5, G=0.0429, \operatorname{Re}=45155$,

(f) $m=4, G=0.0429, \operatorname{Re}=61575$, (g) $m=3, G=0.0714, \operatorname{Re}=49260$,

(h) $m=1, G=0.0714, \operatorname{Re}=102625$, (i) $m=5, G=0.0714, \operatorname{Re}=24630$. 


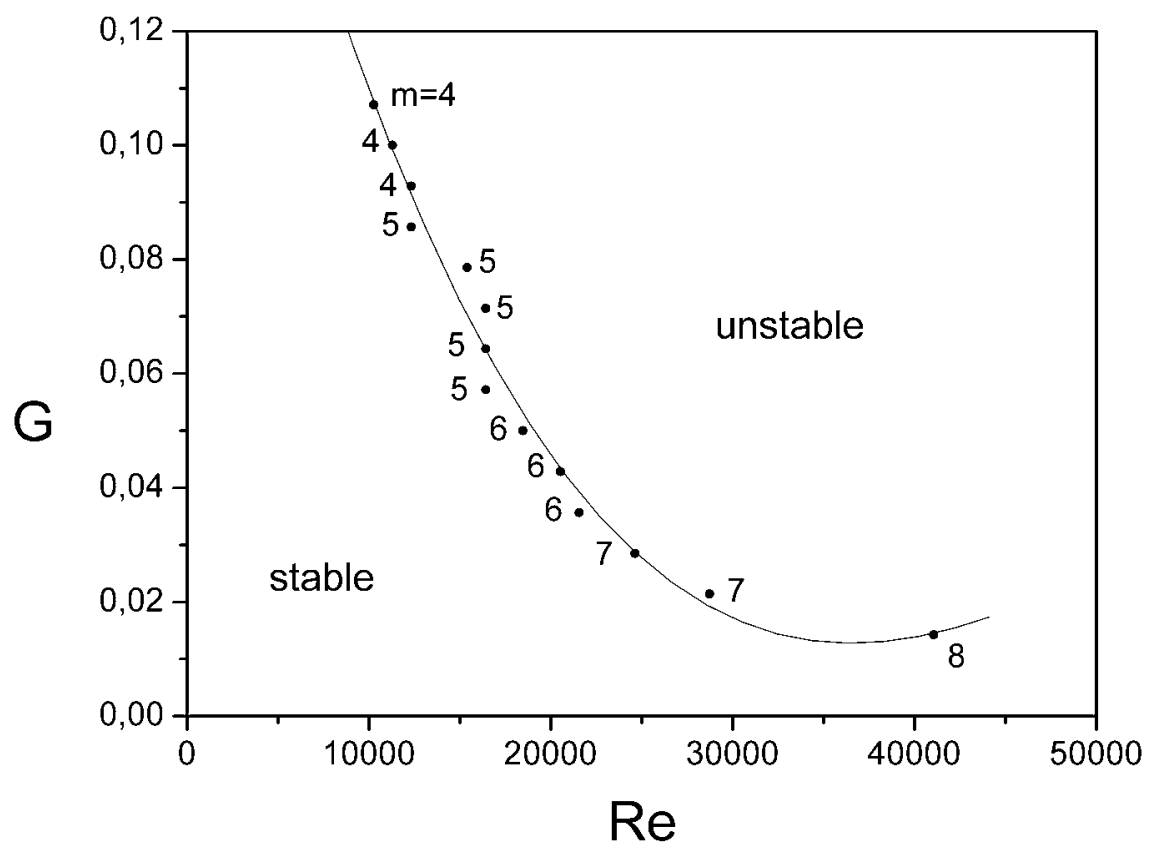

Fig. 7 Marginal stability diagram of the first observed polygonal mode in the plane $(R e, G)$ for $s=0$ and increasing values of $R e$.

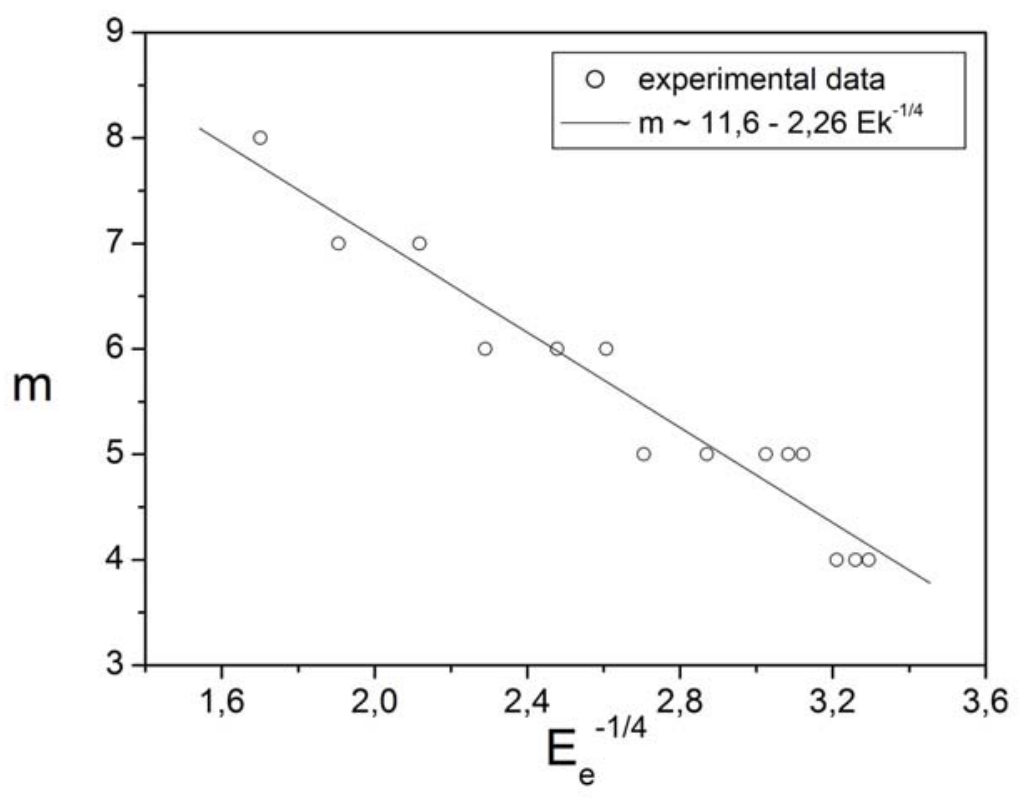

Fig. 8 Evolution of the number of vortices with the Ekman number based on the water depth for $s=0$ and increasing values of $R e$. 


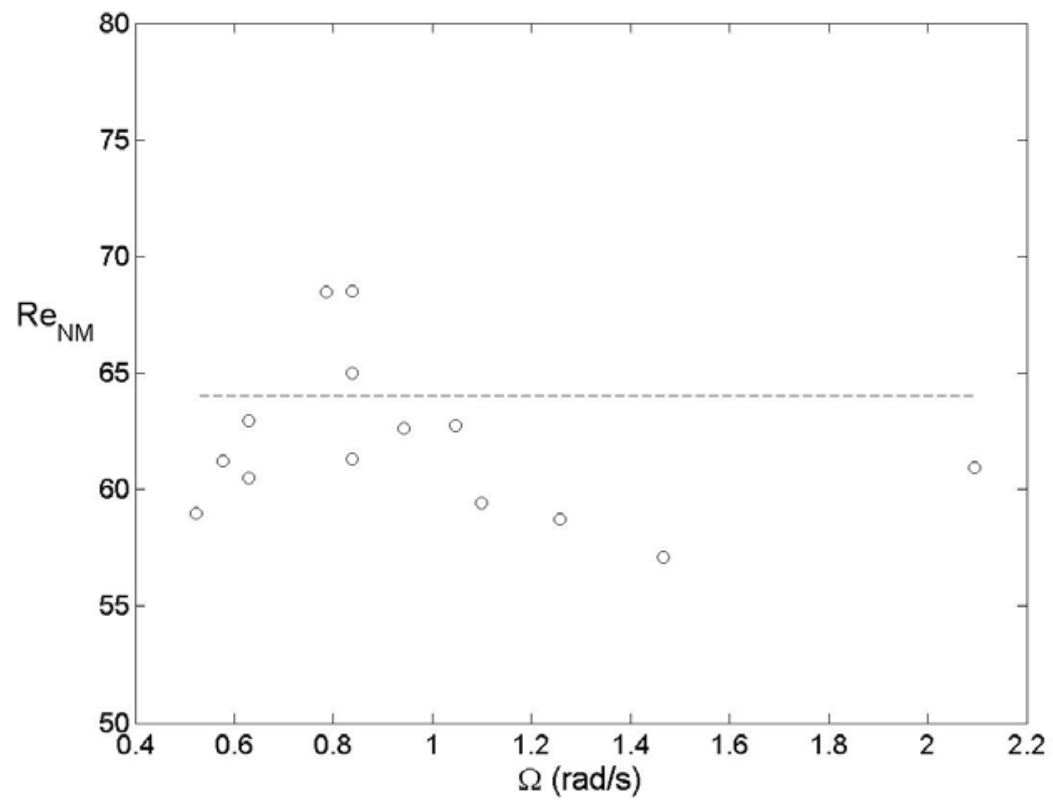

Fig. 9 Evolution of the critical Reynolds number with the rate of rotation for $s=0$.

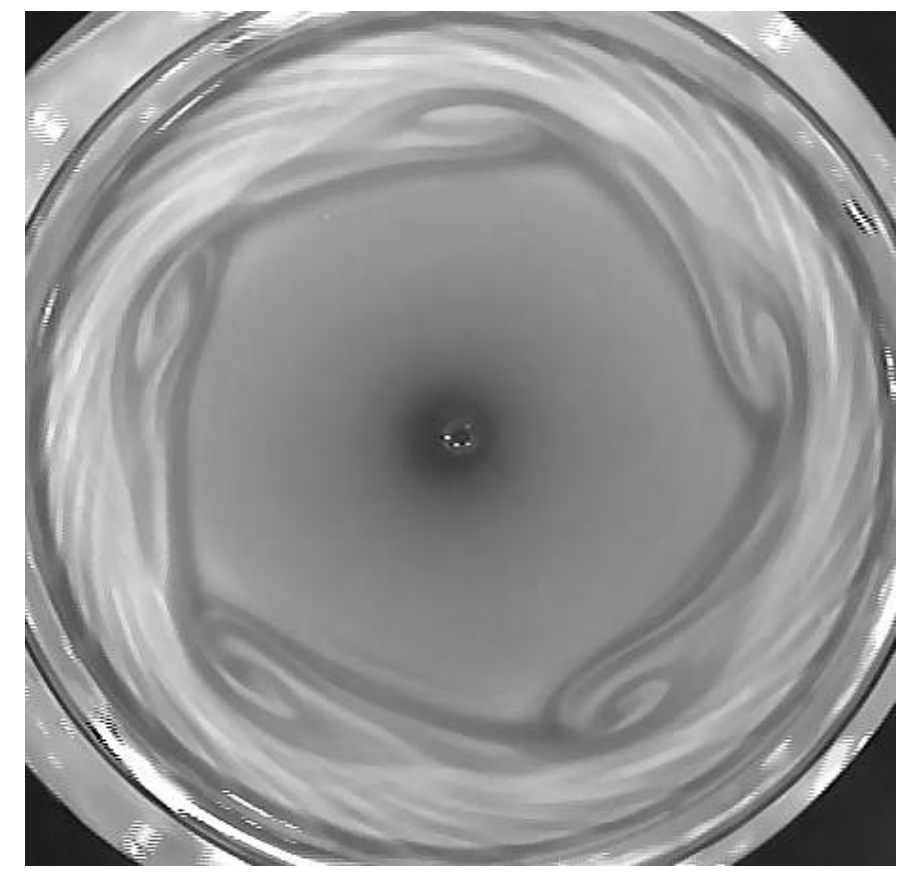

Fig. 10 SRJ2 spiral rolls for $s=0, \operatorname{Re}=24630$ (increasing values of $\Omega$ ) and $G=0.0714$ coexisting with the mode $m=5$. 


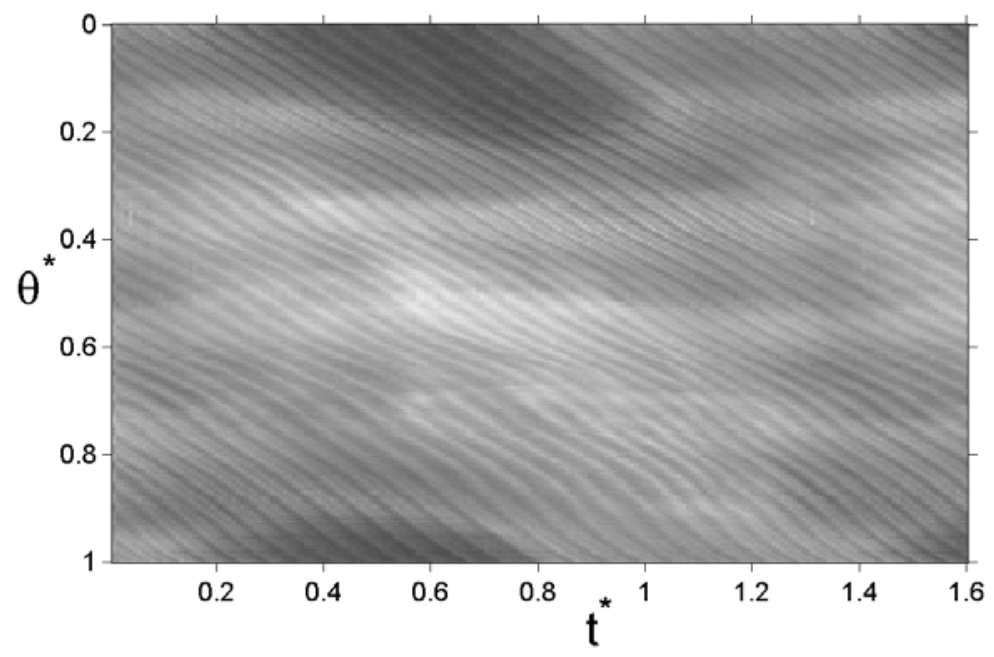

Fig. 11 Space-time diagram for $s=0, \operatorname{Re}=24630$ (spin-up) and $G=0.0714$ in terms of a fixed circle $\left(\theta^{*}\right)$ at $r^{*}=0.95$.

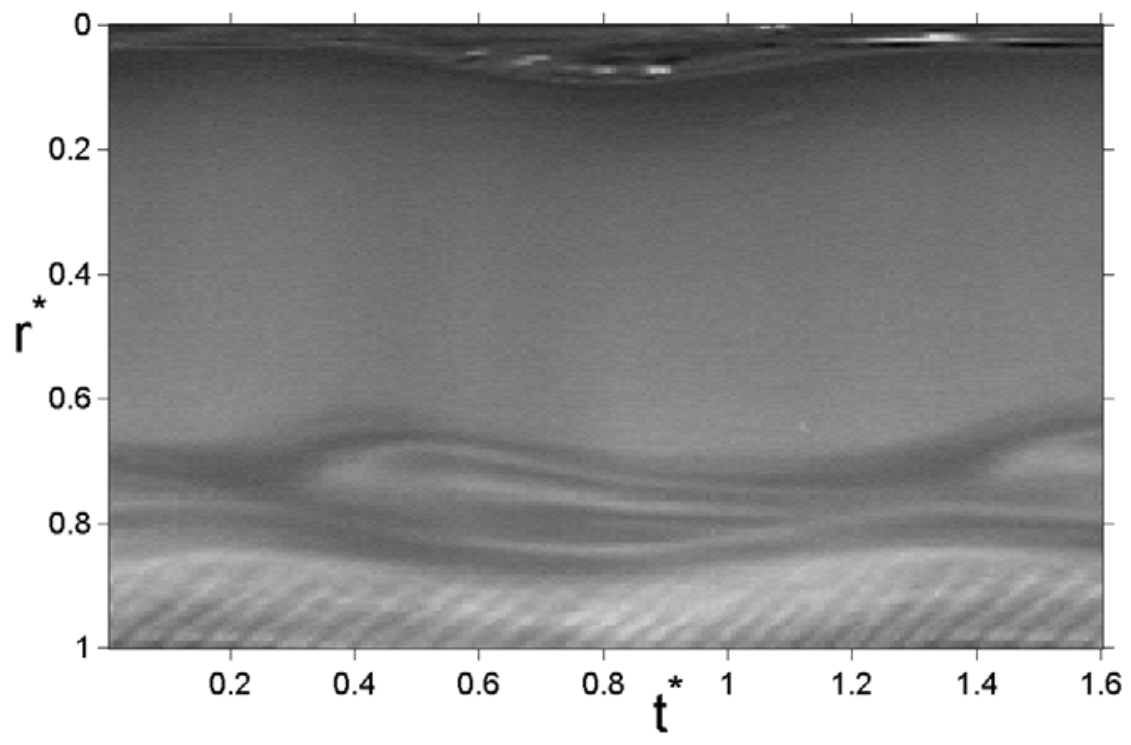

Fig. 12 Space-time diagram for $s=0, \operatorname{Re}=24630$ (spin-up) and $G=0.0714$ in terms of a radius $r^{*}$. 


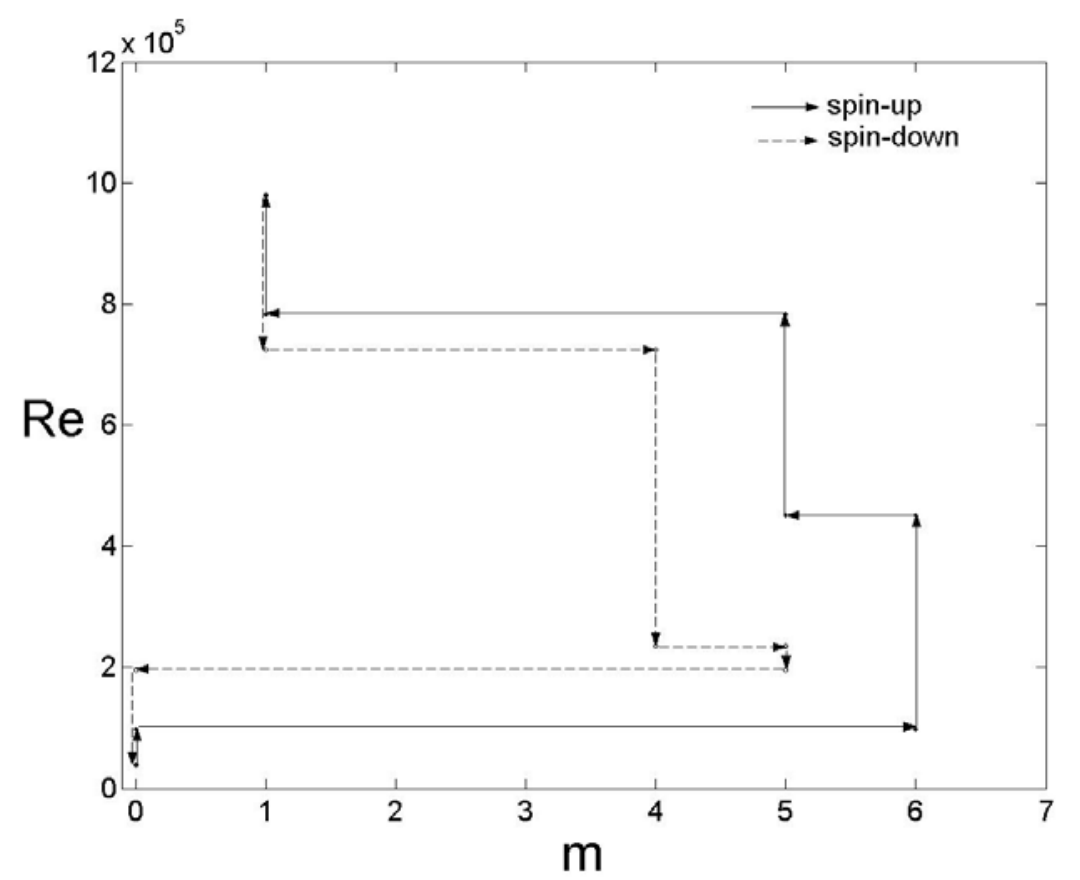

Fig. 13 Stability diagram for $s=0.286$ and $G=0.0429$.

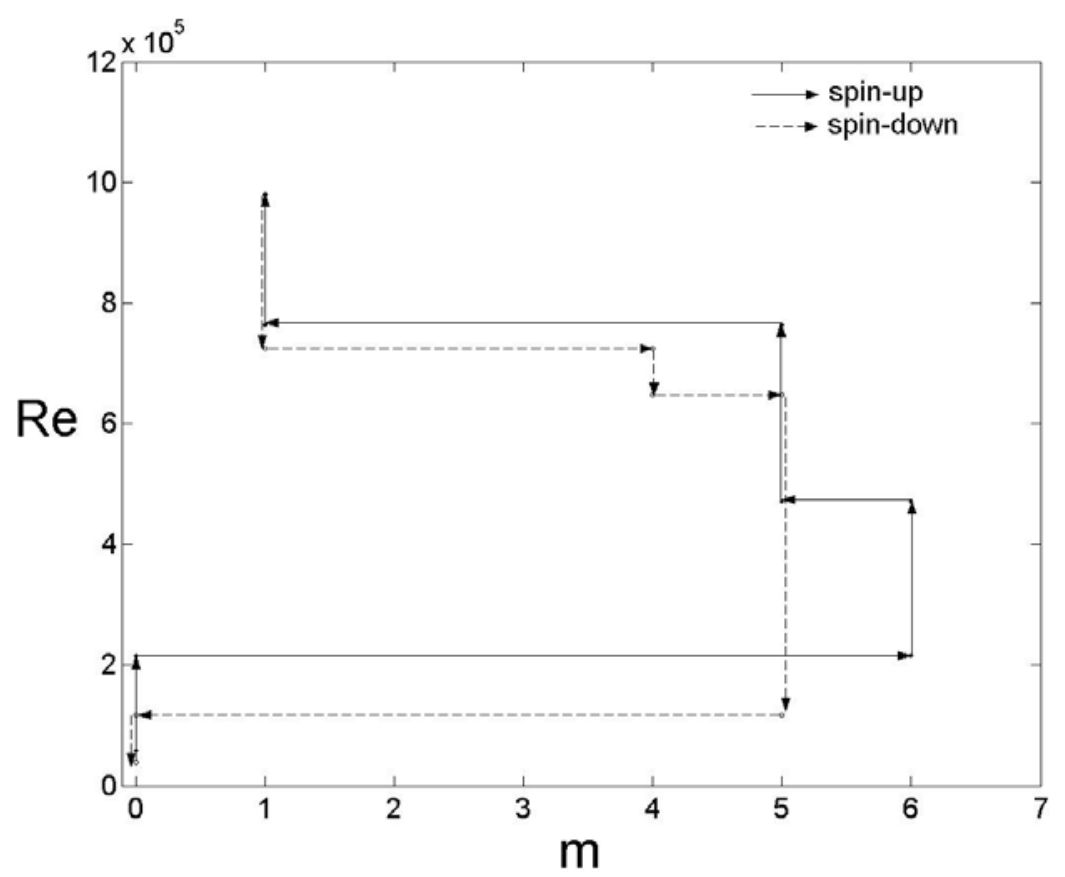

Fig. 14 Stability diagram for $s=0.536$ and $G=0.0429$. 


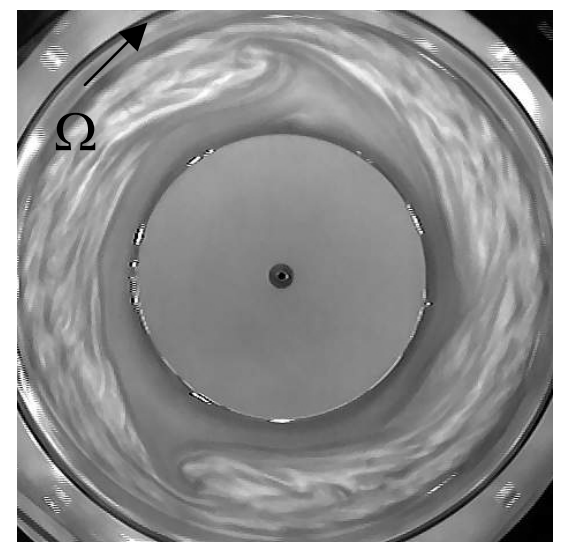

Fig. 15 Mode $m=2$ of the shear-layer instability for $s=0.536, G=0.0714$ and $\operatorname{Re}=41050$ (spin-down)

(a)

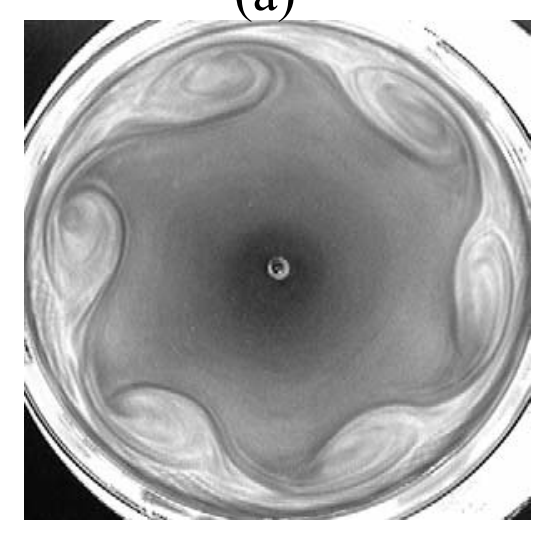

(c)

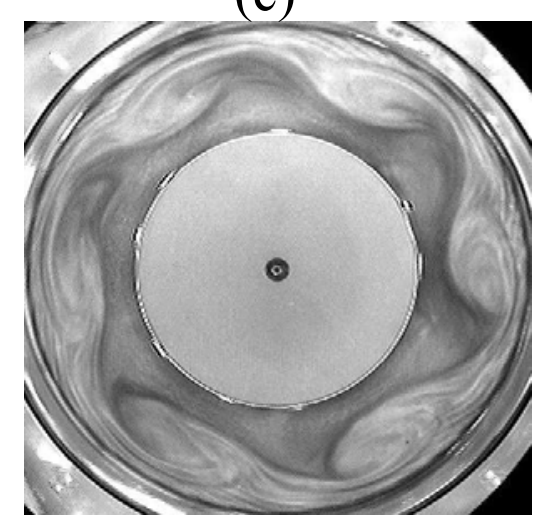

(b)

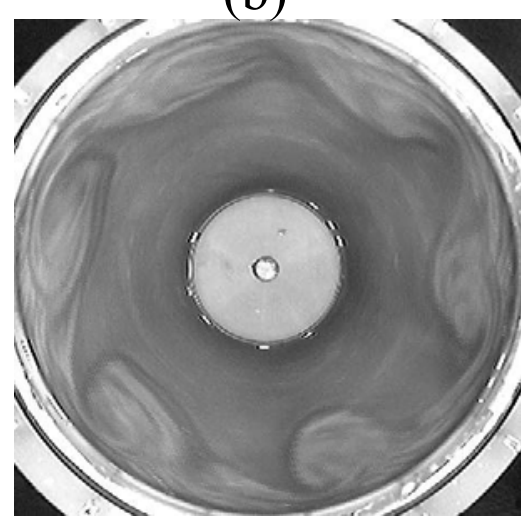

(d)

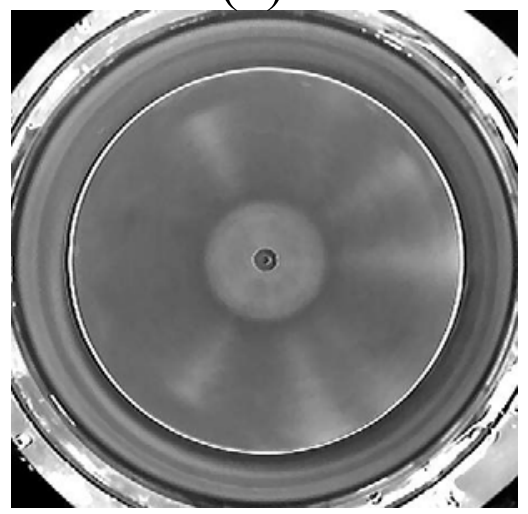

Fig. 16 Influence of the radius ratio $s$ on the flow pattern for $G=0.0429$ and $\mathrm{Re}=36945$ (spin-up): (a) $s=0$, (b) $s=0.286$, (c) $s=0.536$, (d) $s=0.75$. 
(a)

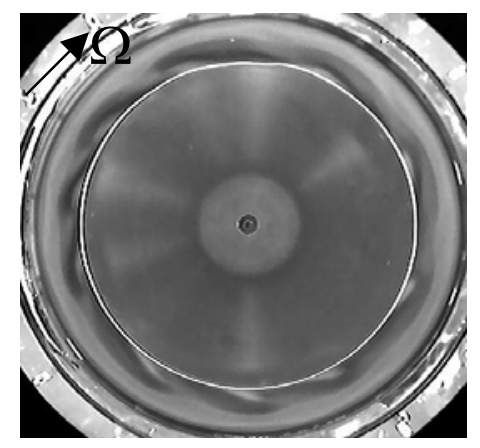

(d)

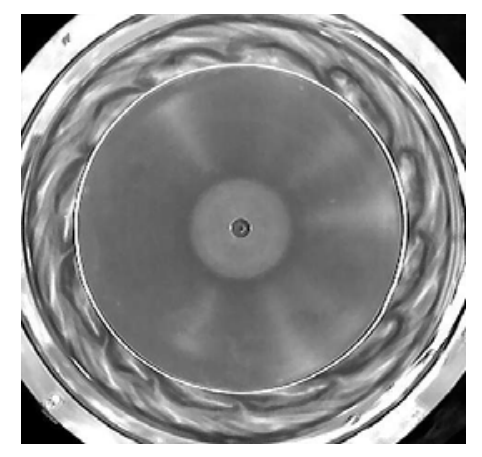

(b)

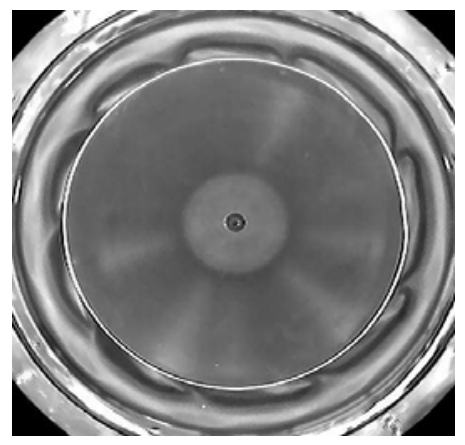

(e)

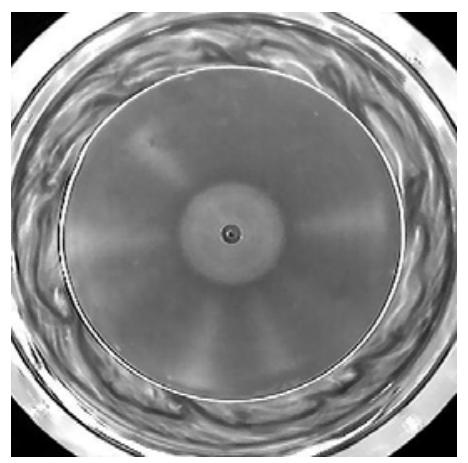

(c)

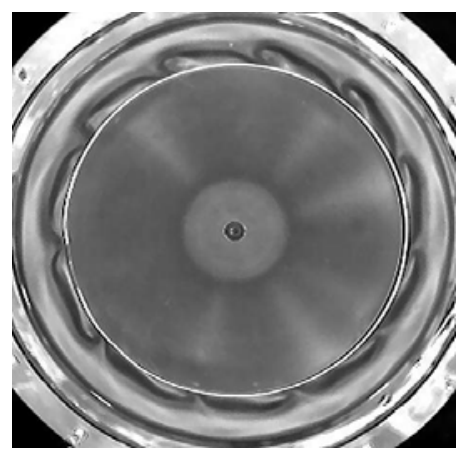

(f)

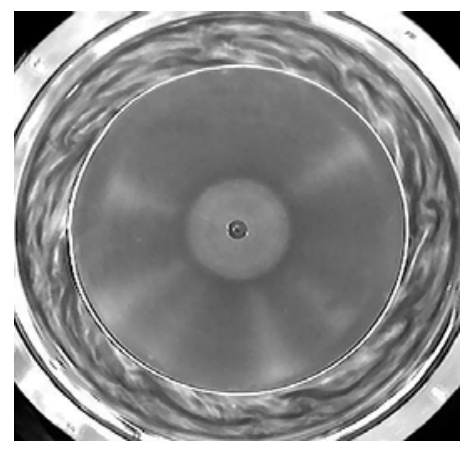

Fig. 17 Shear-layer instabilities on the hub for $s=0.75$ and $G=0.0714$ (spin-up): (a)

$$
\operatorname{Re}=10263 \text {, (b) } \operatorname{Re}=16420 \text {, (c) } \operatorname{Re}=20525 \text {, (d) } \operatorname{Re}=24630 \text {, (e) } \operatorname{Re}=28735 \text {, (f) }
$$

$$
\operatorname{Re}=34893 \text {. }
$$

